\title{
Resin composite contours
}

\author{
H. Sidelsky
}

VERIFIABLE CPD PAPER

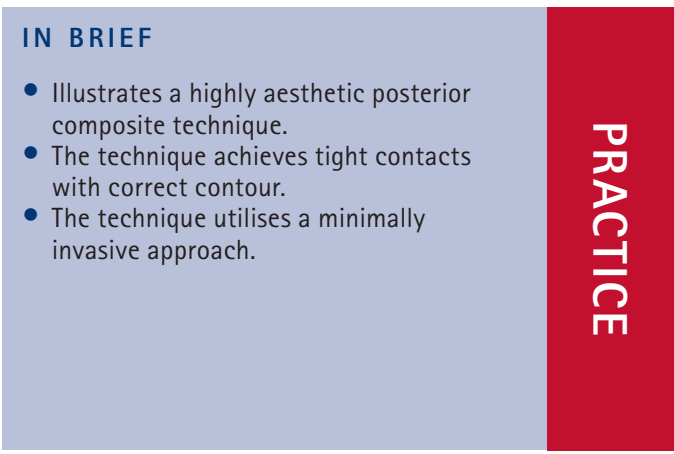

When placing posterior composite resin restorations, clinicians often struggle to achieve good contacts. Frequently contacts that are successful are only confined to the occlusal aspect of the proximal wall. A clinical technique is discussed which achieves the correct contour as well as tight contacts. The technique is also minimally invasive and highly aesthetic.

\section{INTRODUCTION}

In conjunction with bonding agents, tooth coloured restorations have replaced amalgam as the material of choice for many practitioners, for posterior restorations. One dental school has changed from teaching dental amalgams to teaching resin composites exclusively. ${ }^{1}$

Resin composites have the obvious advantage of aesthetics, which also makes them the preferred selection for patients, and through bonding they are able to reinforce undermined cusps, protecting them from fracture to a greater extent than amalgam. ${ }^{2}$

Due to its better mechanical properties resin composite is used rather than glass ionomer on occlusal surfaces.

A literature review was conducted by typing into Medline 'resin composite fillings' and the words 'contact and contour.' Peumans et $a l .{ }^{3}$ found that condensable resin composites do not achieve better contacts than conventional resin composites. Gonzalez Lopez et al. ${ }^{4}$ used an individualised wedge modified with resin to achieve an adequate contact point. They placed a band preoperatively and injected resin composite around a preplaced wedge. The resin composite was cured and withdrawn

2 Devonshire Place, London, W1G 6HJ

Correspondence to: Dr Harris Sidelsky

Email:harris@harrissidelsky.com

\section{Refereed Paper}

Accepted 22 February 2010

DOI: $10.1038 /$ sj.bdj.2010.398

${ }^{\circ}$ British Dental Journal 2010; 208: 395-401 thereby reproducing the shape of the band. Following cavity preparation the band was replaced and the wedge inserted to re-establish the original contour.

El Badrawy et al. ${ }^{5}$ evaluated proximal contacts of posterior resin composite restorations with four placement techniques and found that ceramic inserts resulted in the highest proportion of acceptable contacts. In this study the tightness of the contacts were assessed subjectively by the examiners. No opinion was posited on contour.

Francci et $a l .{ }^{6}$ illustrated a novel technique for using packable composite resins in Class II restorations to achieve contour and contact. They suggested forming the marginal ridge first and then removing the band. No description was postulated on how this achieves the objective of good contour or contact.

Loomans et al. ${ }^{7}$ have carried out many in vitro studies. When looking at the influence of composite resin consistency and placement technique on proximal contact tightness, on class II restorations, they found that the use of a separation ring had a greater influence than the resin composite on contact tightness.

In comparing proximal contact tightness of class II resin composites they found that the use of sectional matrices combined with separation rings provided tighter contacts than circumferential systems. ${ }^{8}$ The same authors also looked at proximal contour, but only on its effect on marginal ridge fracture, and found that the contoured matrix achieved a stronger marginal ridge than the straight matrix. ${ }^{9}$
In another in vitro study they examined the overhangs that were created when using different matrix band systems. ${ }^{10}$ They concluded that circumferential matrices or sectional flexible matrices resulted in the least marginal overhang when combined with a Contact Matrix separation ring or a Composi-Tight Gold ring, but no made reference to contour.

The same investigators found that sectional matrices produced better contacts than circumferential bands in vivo but found that proximal contacts cannot be relied upon to remain stable over time..$^{11,12}$

This review of the literature in this area confirms that research has concentrated around the tightness of the contacts with reduced concern surrounding the contour of the final restoration.

The aim of this article is to describe a clinical technique which can be used in small to medium sized cavities to produce a good contact and contour.

\section{DESCRIPTION OF TECHNIQUE}

In order to assess the extent of the lesion it is essential to take a bitewing radiograph. Rubber dam is placed following local anaesthesia.

The next step is to cut a small piece of matrix band. This should be large enough to extend beyond the buccal and lingual interstitial areas.

The Siqveland matrix band (Dental Directory), more commonly used for amalgam placement, is not as thin as other products but has the advantage of being able to be placed between the two teeth 
without distortion - this is crucial to the success of the technique. A pair of Spencer Wells grips the corner and the band is forced between the two teeth. On the rare occasion where the contact is too tight a wedge can first be placed to separate the two teeth (Fig. 1). After the band is in place an anatomically shaped wedge (Cardoza Dental) is placed between the teeth (Fig. 2). This has the effect of ensuring there is no gap between the band and the tooth cervically and at the same time it forces the adjacent tooth slightly further away from the prepared tooth. It also holds the band in a firm position.

The end of the wedge is shortened by breaking off the edge to allow us to place a Palodent clamp (Optident UK). If the wedge is inserted at its original length, it might jut out and prevent the clamp from engaging. The Palodent clamp is then placed taking care to engage the teeth (Fig. 3). Placement of the band as the first step is crucial to the technique as it allows the band to form around the contour of the existing intact approximal wall. Following the cavity preparation the shape of the band is preserved due to its stiffness and the firmness of the contact is further facilitated by the Palodent clamp.

Figures $4 \mathrm{a}$ and $4 \mathrm{~b}$ show the initial occlusal access cavity. This is placed approximately $1 \mathrm{~mm}$ away for the edge of the marginal ridge into the fossa. The bur usually plunges into the lesion and with magnification one can assess the amount of caries. Figures 5a and 5b show a very common presentation of an approximal wall view of the shape of many interstitial carious cavities. If the cavity is shaped like Figure $5 b$ then the bur is moved buccolingualy enlarging the access hole as necessary to allow further visualisation. This facilitates removal of the caries. At the same time one should try and do this by preserving the maximum amount of tooth substance (Fig. 6). If the access cavity resembles the illustration in Figure 5a then a more limited widening is required.

The caries is removed with a stainless steel round number 2 or 4 bur. The cavity is then washed and dried. At this stage gross caries removal has taken place but obviously it can be difficult to see the entire extent of the lesion interstitially. In the past this has often been the failure of the 'tunnel' preparation. The next step is to

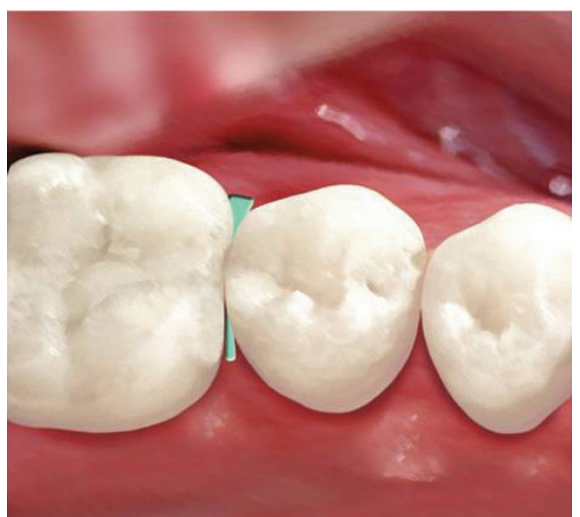

Fig. 1 An anatomical wedge can be used to facilitate band placement

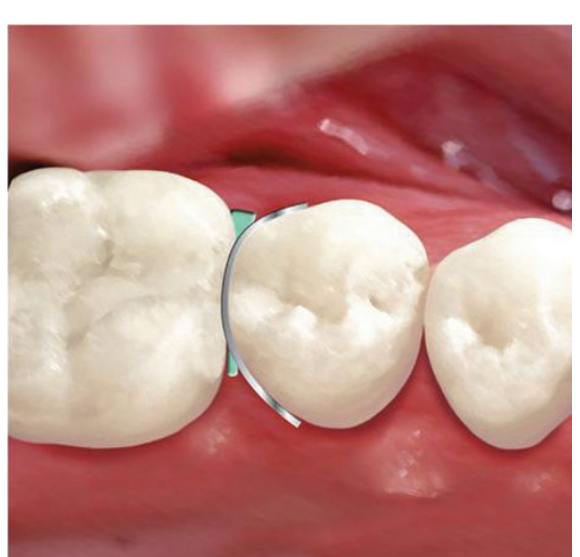

Fig. 2 The matrix band and anatomical wedge are placed

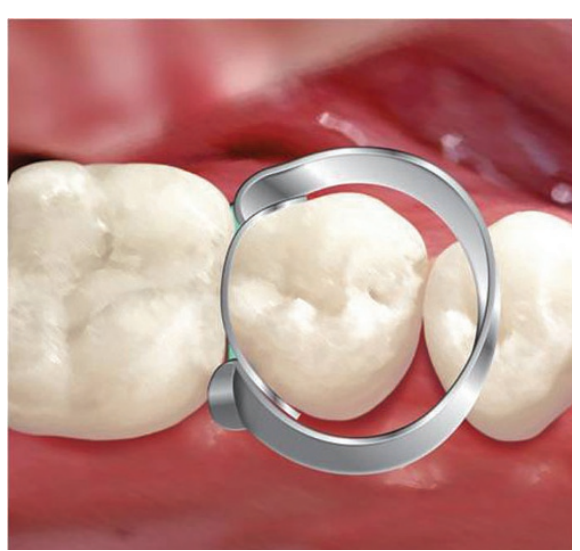

Fig. 3 The palodent clamp is placed

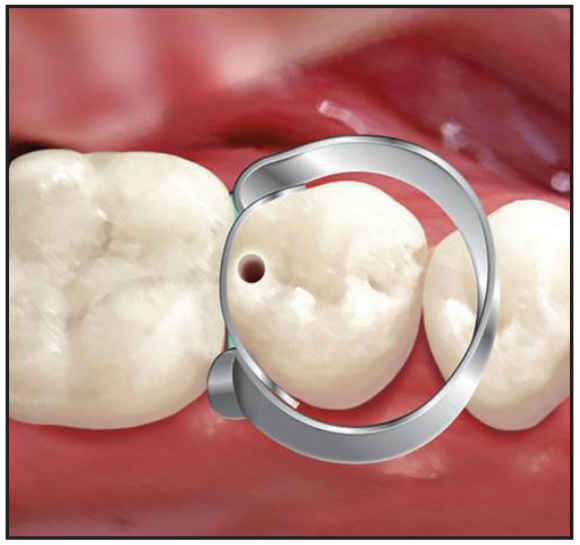

Fig. 4a A small access whole is drilled

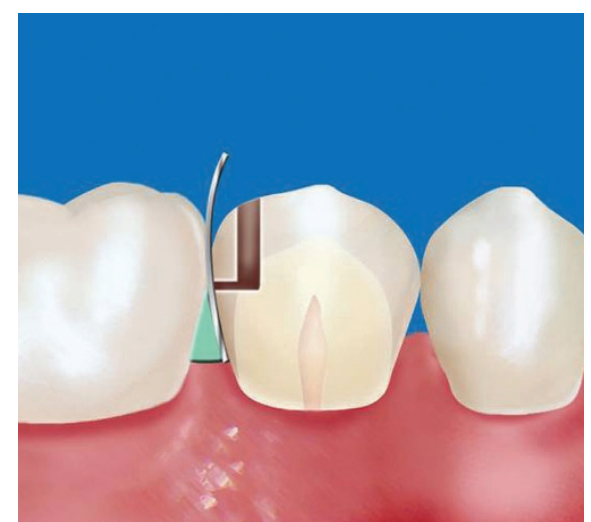

Fig. $4 \mathrm{~b}$ Saggital view of the access hole

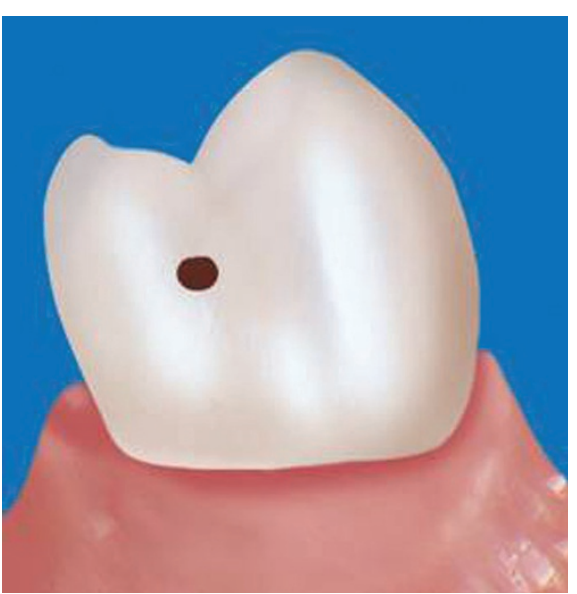

Fig. 5a The access hole can be round

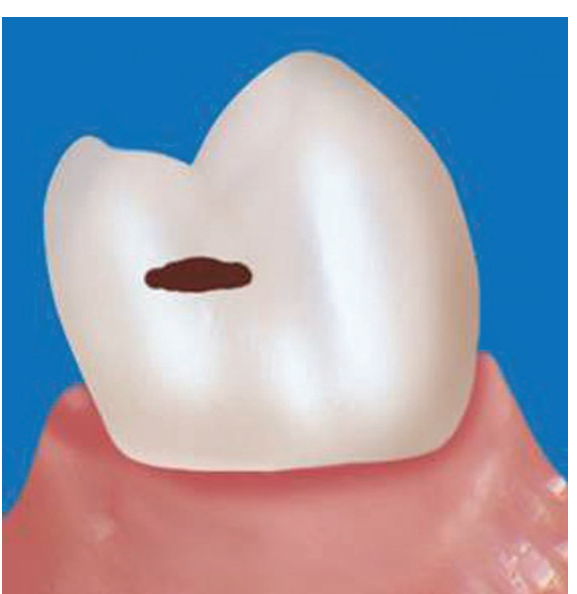

Fig. 5b The access hole can be rectangular

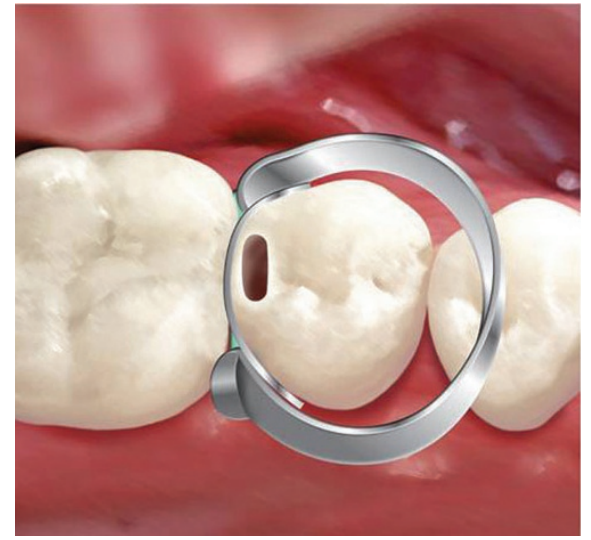

Fig. 6 The access hole is widened 


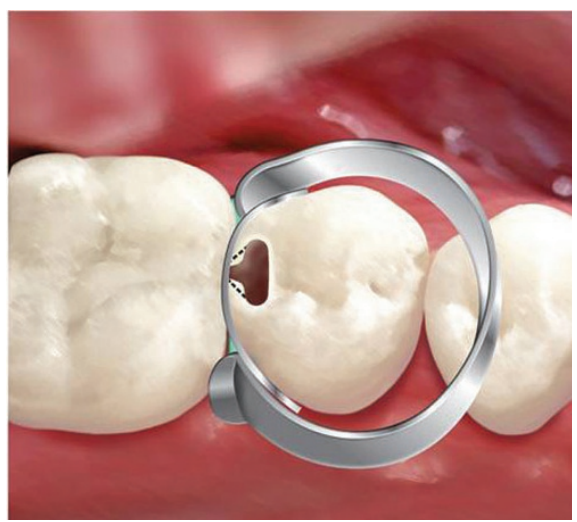

Fig. 7 The marginal ridge above the cavity is carefully thinned and then broken

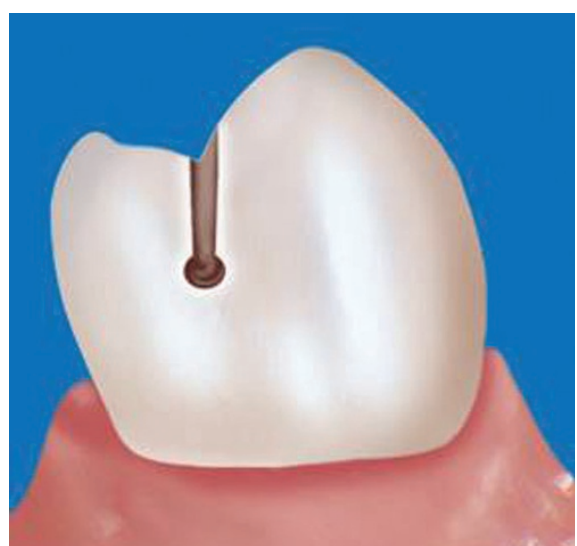

Fig. 8 Typical appearance of prepared cavity shape for when original cavity is round

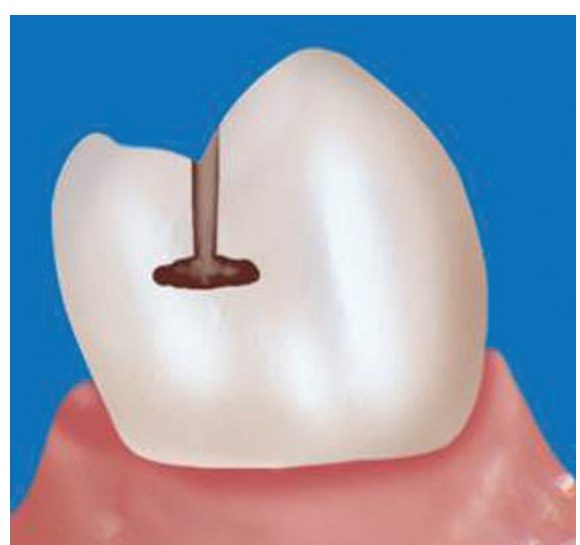

Fig. 9a Appearance prior to modification

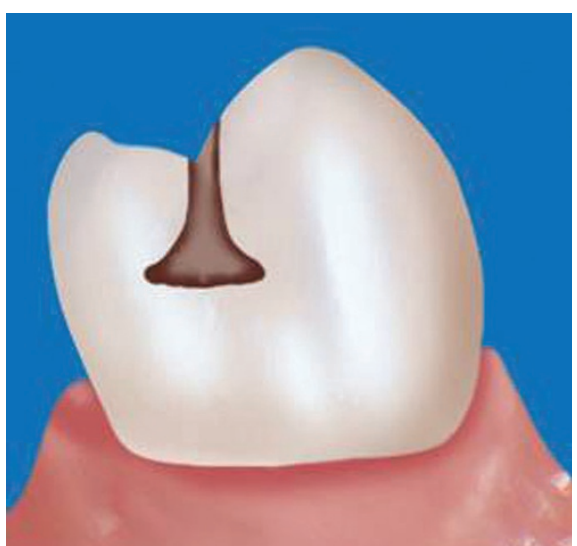

Fig. 9b Modified cavity

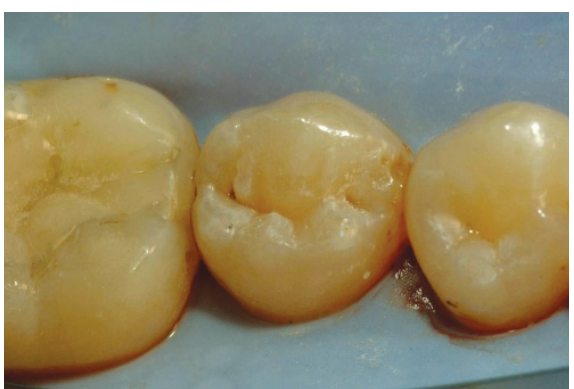

Fig. 10 The tooth prior to restoration

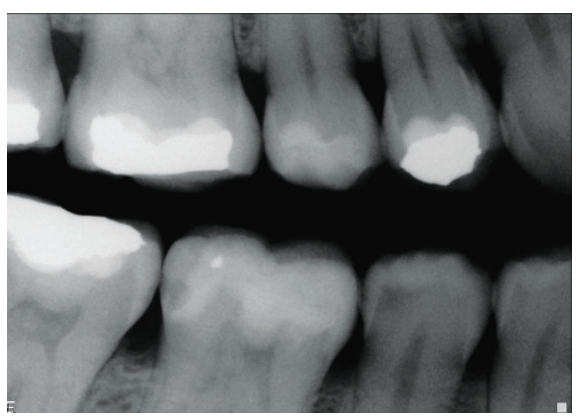

Fig. 11 Radiograph of distal caries in tooth 45

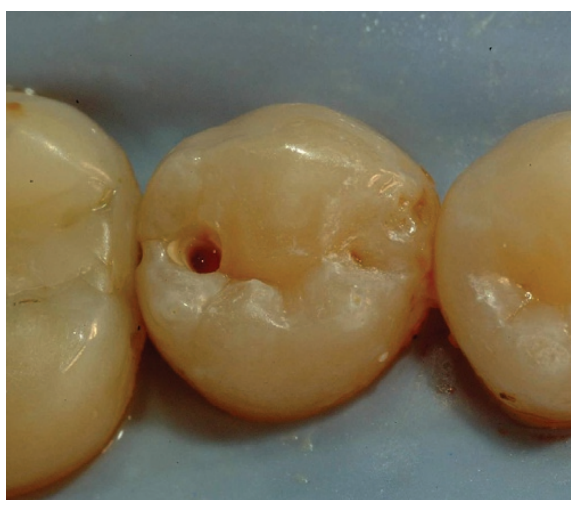

Fig. 12 The occlusal access cavity is prepared

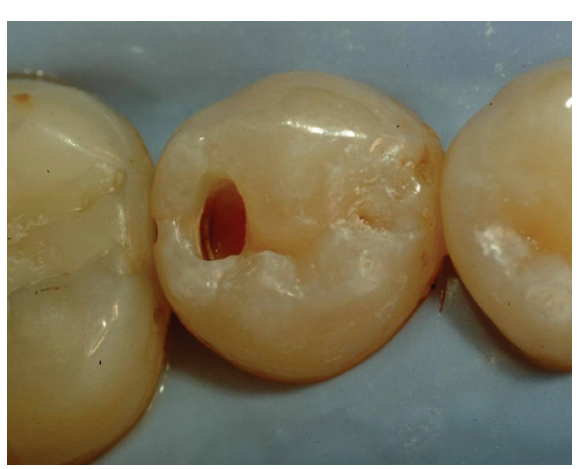

Fig. 13 The cavity is broadened to gain access to caries

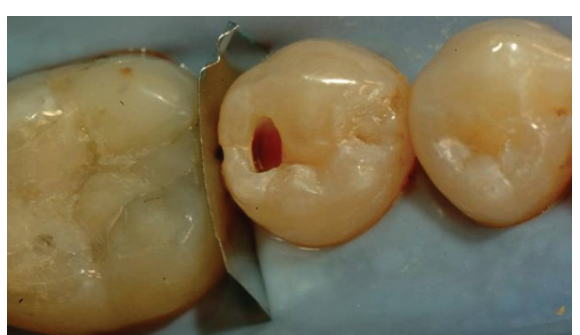

Fig. 14a The section of matrix band is placed and wedged into position use a tapered diamond bur (Heraeus Kulzer bur no 171-016C, Dental Directory) and carefully remove only the marginal ridge area above the interstitial cavity. Figure 7 illustrates the ensuing cavity shape.

If the interstitial cavity is circular (Fig. 5a), then only the marginal ridge above this cavity is removed. One can often thin the ridge and then the final removal takes place by breaking the undermined piece. This will create a cavity shape, illustrated in Figure 8. If the interstitial cavity is rectangular shaped (Fig. 5b), then the cavity is extended by joining the marginal ridge to the outer point of the rectangle on the proximal wall. Figures 9a and 9b show how the cavity may look before and after this step. This effectively creates a cantilever of the marginal ridge. Every effort is made to preserve as much of the marginal ridge as possible. Where it is extremely thin then that section of the ridge may be removed but in most cases this is not the case as the caries has not penetrated to this area.

It will now be possible to see and remove all the caries in the cavity using magnification. Obviously this would only include peripheral caries and infected caries but not 'affected caries' over the pulp. As long as the demineralisation process has not created complete destruction of tooth form one can often remineralise the affected dentine. ${ }^{13,14}$

The tooth is then washed well and etched for ten seconds with orthophosphoric acid. (Orthophosphoric acid Bisco etch 37\%, Optident UK) which is then washed off and the tooth is dried. The final appearance of the dentine is crucial and should not be too wet or dry. With this in mind it is usual to gross dry and then useful to use a paper point to carefully remove the excess water. The final appearance of the dentine should resemble 'a thumb nail which has been licked' (author's observation).

A resin modified glass ionomer such as Vitrebond, (now known as Vitrebond Plus) (Espe Dental), is then carefully placed with a suitable applicator over the exposed dentine and set with the curing light.

The whole cavity is then coated with a bonding agent. Optibond Solo Plus (KerrHawe UK) is used in case there are any areas of dentine not adequately covered.

This is cured for 20 seconds. The resin composite is then built up to restore the cavity. 


\section{CASE PRESENTATION}

All procedures are carried out under rubber dam. Figure 10 shows the tooth before restorative care. Figure 11 shows the radiograph of the tooth with caries distal in tooth 45. A small access hole is created in the occlusal surface until the bur penetrates into the caries (Fig. 12). The cavity is enlarged according to the clinical findings with every attempt made to keep the size minimally. If the caries extends towards the mesial then the cavity is enlarged somewhat in that direction. Figure 13 shows the cavity which has in this case been extended more buccolingually. Magnification is used to see the extent of the lesion throughout the procedure.

A section of a Siqveland molar matrix band is passed through the contacts. This can be done before even placing the access hole. It is easier to place it by holding it with a pair of Spencer Wells Artery Forceps. Note that the band should not be too wide and should be cut to width so that it just goes out beyond the outer limit of the tooth in the buccal and lingual direction (Figs 14a and 14b). An anatomical wedge is placed after it has been reduced in size. The largest wedge possible is used. The reason for reducing the size is to allow the placement of the Palodent clamp. If it were to protrude this would not allow for the placement of the clamp in the most effective way. Figure 15 illustrates the Palodent clamp in situ. It is important that the clamp engages the tooth being prepared and the adjacent tooth on the buccal and the lingual side. The band is then checked to make sure there has been no distortion which in this case is rare because of the sequence carried out and described. The next step is to carefully thin the marginal ridge above the interstitial cavity. The thinned out section can then be broken off by using a PK Thomas 3 instrument (PK Thomas number 3 wax instrument - www.hufriedy.de) (Figs 16 and 17). It will now be possible to see clearly into the cavity and once this visual access has been gained the next step is to remove all the caries. This is accomplished by using a number two, three or four round tungsten carbide bur in a slow handpiece. If the original cavity was of the rectangular shape (Fig. 5b) then a small excavator can be used to scoop out the caries. One may have to modify

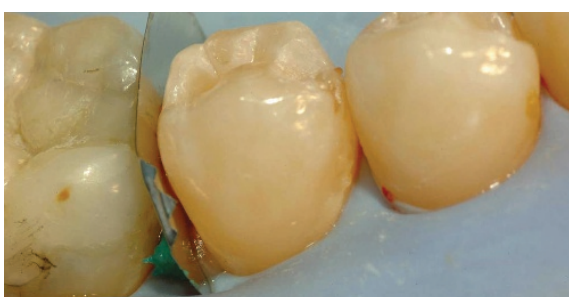

Fig. 14b The anatomical wedge is reduced in size

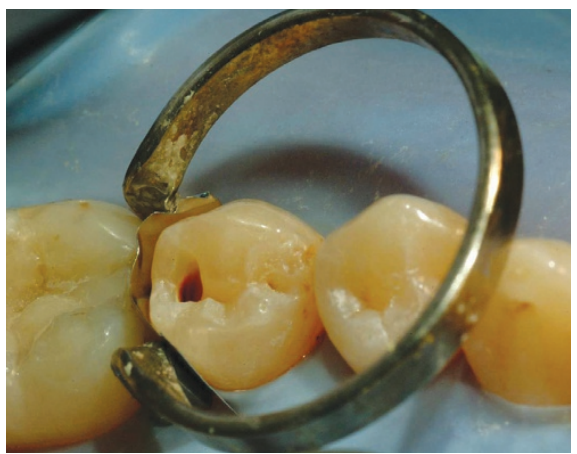

Fig. 15 The palodent clamp engages the two teeth

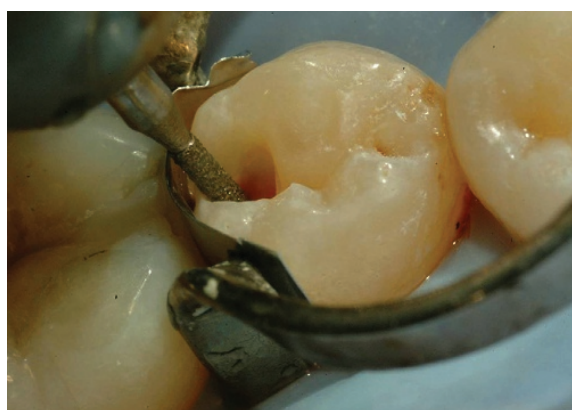

Fig. 16 The tooth is thinned above the initial cavity

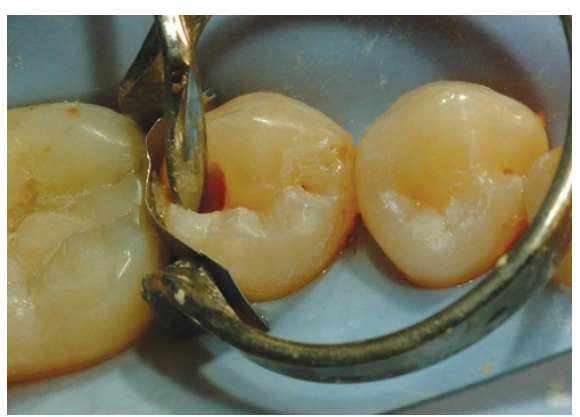

Fig. 17 The thinned wall can be broken with a PK Thomas 3 instrument

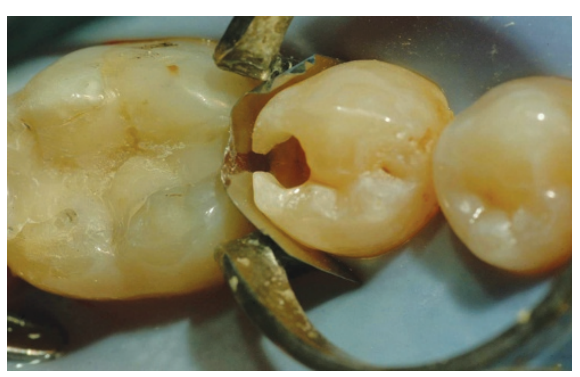

Fig. 18 Visual access has been gained with minimal damage to the wall

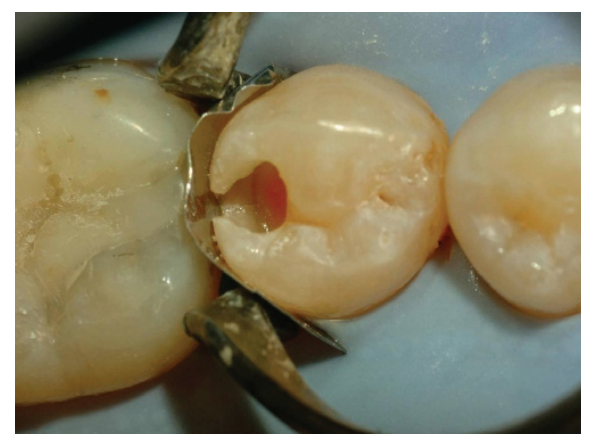

Fig. 19 The caries is removed

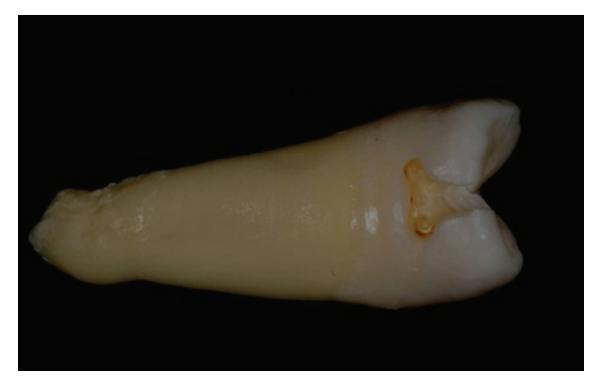

Fig. 20 Common completed cavity shape when initial lesion is rectangular

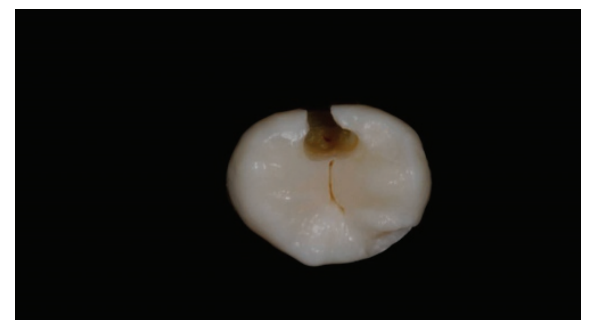

Fig. 21 Common occlusal view of completed cavity

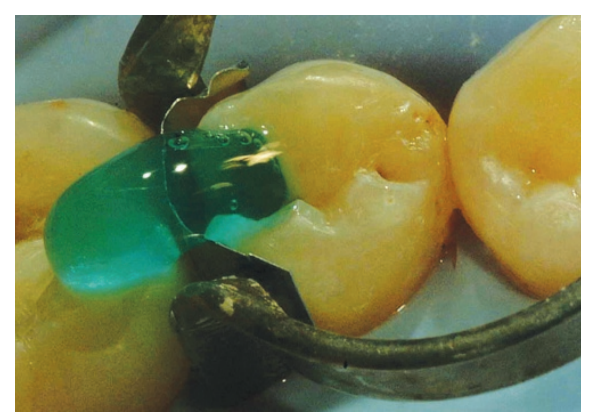

Fig. 22 The tooth is etched

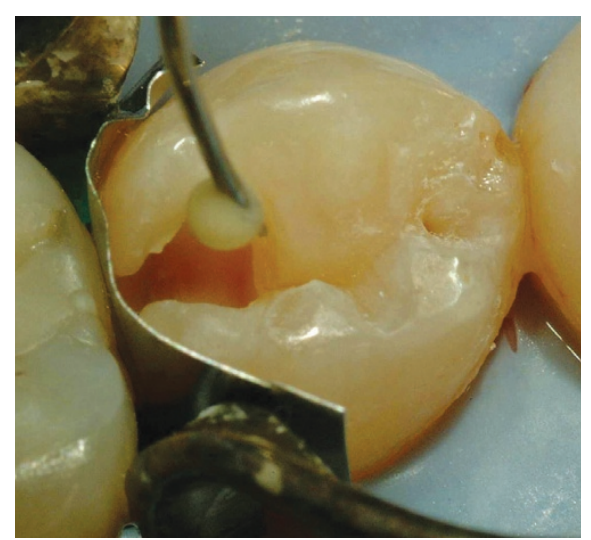

Fig. 23 Vitrabond is applied over the dentine 


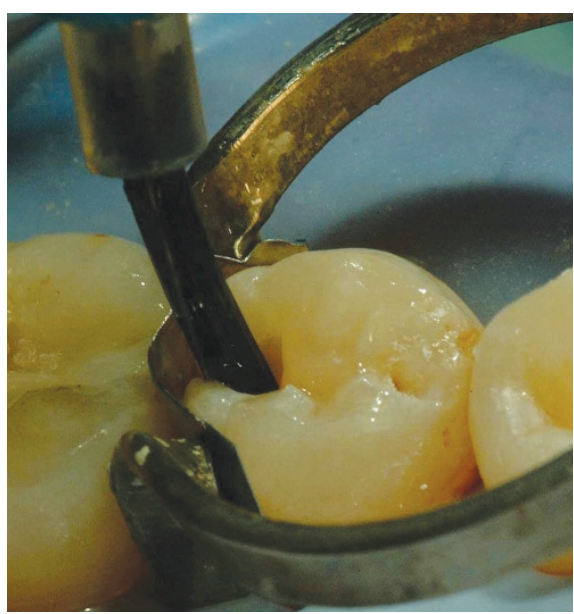

Fig. 24 Bonding agent coats the cavity

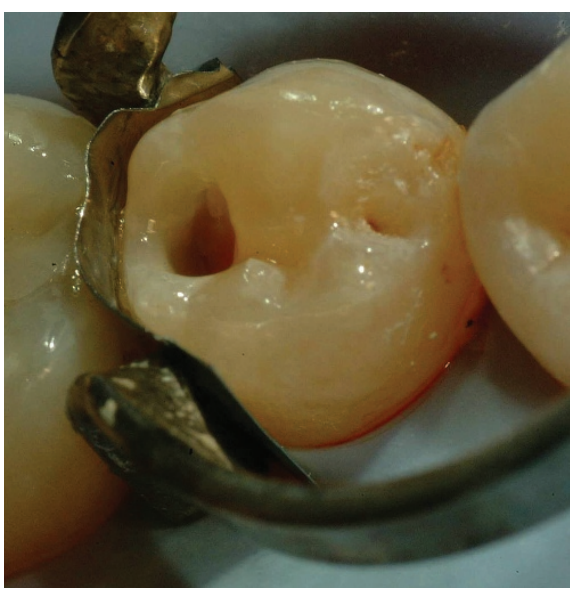

Fig. 25 The marginal ridge is built

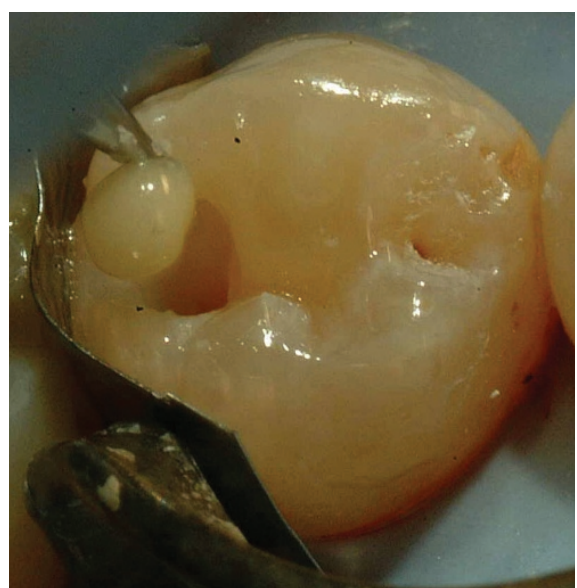

Fig. 26 The cavity is filled incrementally

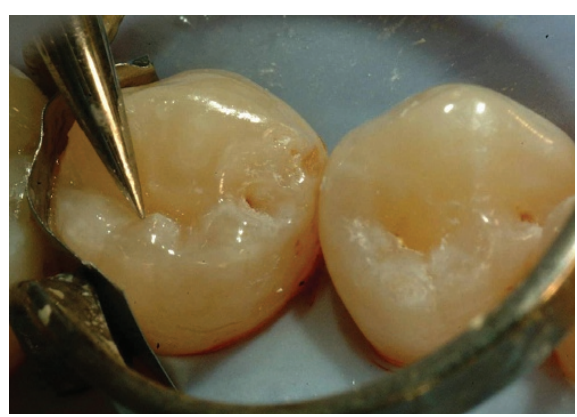

Fig. 27 The final shape is created using a PK Thomas 3 instrument

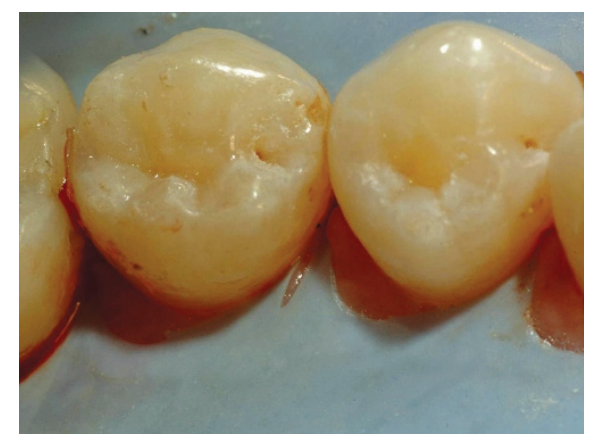

Fig. 28 The completed restoration is aesthetically successful

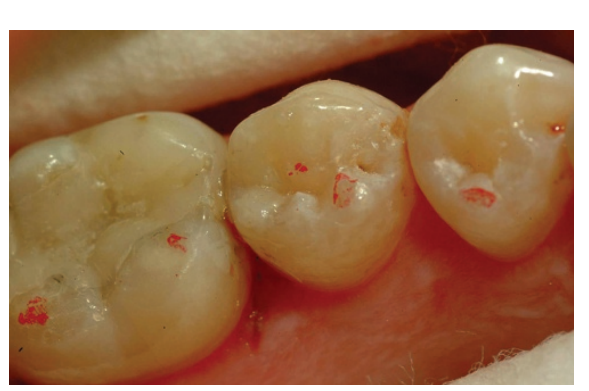

Fig. 29 The rubber dam is removed and the occlusion checked

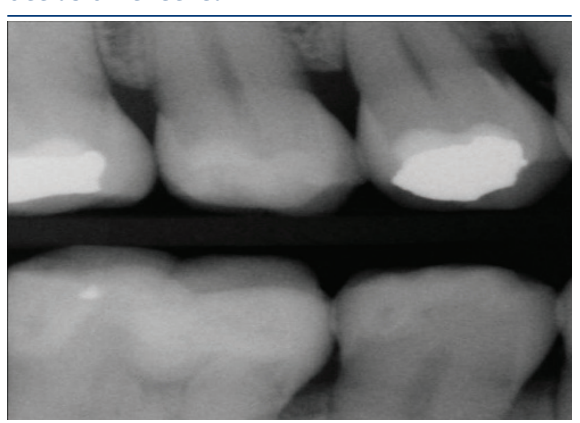

Fig. 30 Post-operative radiograph

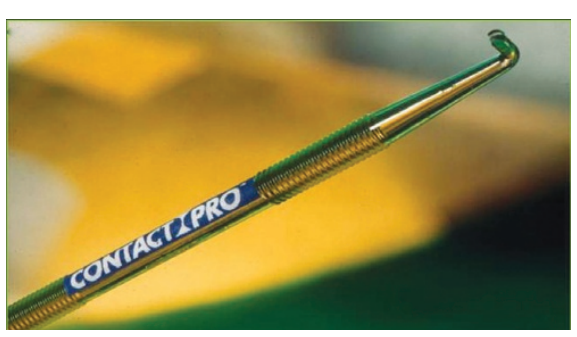

Fig. 31 The contact pro instrument attempts to create contour and contact

the shape of the cavity to gain access to the caries. Again every attempt is made not to remove more marginal ridge than is necessary and this is crucial in maintaining contour. Magnification is essential and the whole cavity must be explored. Figures 18 and 19 show the cavity prepared and one can see most of the marginal ridge is still intact. Figures 20 and 21 show an extracted tooth to enable one to visualise what the end result often looks like - this view not being possible in our case presentation.
Once the cavity has been rendered 'infected caries free' (one often leaves 'affected' caries on the axial wall) the next step is to restore the tooth. ${ }^{13,14}$

The tooth is etched (Fig. 22). The etch is removed by copious washing and the excess water removed leaving the dentine moist but not wet. Vitrebond, now known as Vitrebond Plus, is applied to the exposed dentine and cured (Fig. 23). The whole cavity is then covered with a bonding agent, Optibond Solo Plus, and this is then dried and cured (Fig. 24). The marginal ridge is then built up using generic enamel (Enamel plus, Optident UK) (Fig. 25). The rest of the cavity is then incrementally built up taking into account the ' $\mathrm{C}$ ' factor by placing and curing a small piece of resin at a time (Fig. 26). ${ }^{15}$ This ensures the relief of polymerisation stress by permitting the shrinkage of composite resin into the open space adjacent to the placed increment. The ' $\mathrm{C}$ ' factor can be formulated by dividing the bonded surfaces by the unbonded surfaces and therefore smaller increments ensure that this score is low. As well as reducing sensitivity it also allows the placement of the anatomy and colour. Figure 27 shows how one uses the PK Thomas number three instrument (HuFriedy) to develop the final anatomy.

Figure 28 shows the final restoration before the rubber dam being removed and Figure 29 shows the occlusion being checked following removal of the rubber dam. Figure 30 is a bitewing radiograph taken at a routine follow up examination two years after the initial restoration was completed.

\section{DISCUSSION}

Every practitioner is clinically aware of the problem of establishing a good contact and contour in direct resin composites. These resin composites provide very little internal force to counter the force applied by the matrix band, unlike amalgams which possess a high force to resist deformation. This has generated a plethora of techniques and equipment to help overcome this difficulty.

The tunnel technique attempts to preserve the marginal ridge and axial shape in using a minimal invasive approach. ${ }^{16}$ Its outstanding aesthetics have made it a popular approach but considerable doubts have emerged in the literature concerning 
its success. ${ }^{17-20}$ These have centred around recurrent caries at the approximal site and post placement fracturing marginal ridges. The slot prep was compared and found to be a more reliable alternative. ${ }^{20}$

When an amalgam is placed the matrix band is removed before the material setting and the restoration springs back and establishes a good contact. Unfortunately resin composite is set before the band removal and often leaves a gap between the new restoration and the adjacent tooth. To overcome this difficulty one can 'prewedge' and separate the two teeth. Following placement of the restoration the teeth spring back together closing the space when the band and wedge are removed. Alternatively at the time of placement one can apply pressure against the band to force it against the adjacent tooth. This has given birth to instruments such as the 'Contactpro' (V Ring sectional matrix, Triodent) (Fig. 31).

Many of these techniques at best often establish a good contact at the marginal ridge area but the approximal wall which has been built up often lacks contour. Figures $32 \mathrm{a}$ and $32 \mathrm{~b}$ are diagrams from Wheeler's textbook of dental anatomy. ${ }^{23}$ In Figure 32a one can see from the sagittal view that from the cervical ridge the tooth curves outwards one millimetre to the contact area and then returns along another curve backwards another $1 \mathrm{~mm}$. Figure 32b illustrates the same curve when viewed from the coronal plane. These are arcs and not straight lines. The more tooth substance that is lost cervically the greater the problem of restoring the curve of the contour is accentuated. When matrix bands are placed and the restoration built up it is not uncommon to get a result illustrated in Figure 33. When you have a deep cavity the natural tooth tapers and therefore accentuates the curve that is required to be restored. It is very difficult to seal the matrix band against the floor with a wedge and expect the band to then emerge from the floor to the marginal ridge with a good contour. The red line in Figure 33 shows a very common end result with a straight rather than curved interstitial wall. We see after a lot of prewedging contact is often established at the marginal ridge area but the wall lacks contour. When the floss is passed through the contact after placement the 'click or snap sound and feel' is often missing. Rather the floss is tight at the ridge areas then breaks through into space below. This is not so easy to describe but well known to all practitioners who place resin composite resin restorations and feel the difference in contact from that which is often achieved with a cast laboratory restoration, where contact and contour can be built on a model.

In an attempt to solve this difficult clinical situation a plethora of different bands such as the $\mathrm{V}$ ring (Contactpro CEJ Dental, www.cejdental.com), the Palodent matrix, and the compositight (tight silver plus Garrison dental solutions www.garrisondental.com) have entered the dental world. These have been supported but hard clinical evidence is lacking. ${ }^{21,22}$

Some of these are successful but the clinical consistency is often absent. It is often difficult to insert the matrix bands without altering its shape and frequently burnishing is required to contact the adjacent tooth.

This is especially true when the preparation is minimal. Another scenario is when there has been excessive loss of tooth in the mesial or distal areas. This often neutralises the effect of the Palodent clamps and similar products as they rely on engaging the approximal areas to create separation.

The ideal situation would be to replace the original contour of the tooth. While the technique described in this article has not yet been backed up with any clinical research its approach and clinical result experienced by the author support its use. The principal logic behind the technique is to replicate the contour of the tooth before beginning cavity preparation. This is done by first using a thicker matrix rather than the thin variety as this is less likely to distort following preparation. All efforts are then directed to a minimal invasive technique to preserve this contour. The existing contour of the tooth is preserved and the small void created is filled in with resin composite which bonds together the two sides as well as supporting the remaining wall internally. When post-operative radiographs are taken at follow up routine examinations they consistently reveal a satisfactory contour and this is consistently confirmed clinically with 'the snap' contact when floss is passed through. This is facilitated by the fact that the original
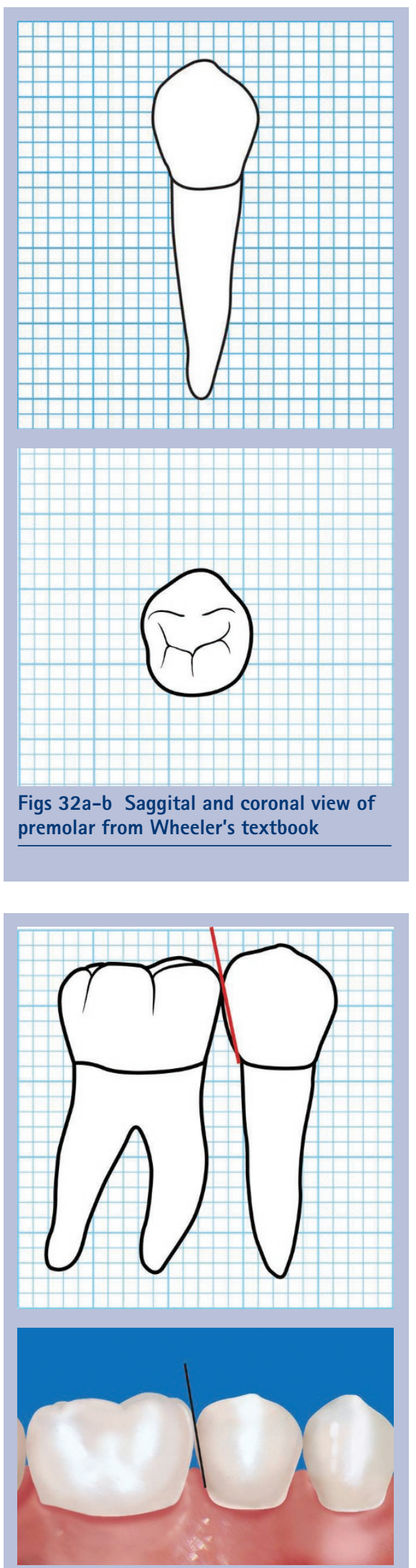

Figs 33a-b The red line indicates a common end product which lacks contour

wall contour is largely preserved through this approach. Although previous studies have confirmed that with the tunnel preparation marginal ridge fracture is a common 
failing, this has not yet occurred on any of the hundreds of restorations placed by the author, who hopes to publish these in vivo findings in the future. In addition the repair to a fractured marginal ridge is a quick, inexpensive and simple procedure. The worst failing with the tunnel technique was secondary caries and this is certainly overcome by virtue of the excellent vision into the cavity described in this article which is often not possible in the tunnel technique. Failure in the latter often occurs due to lack of the filling material penetrating into the original interstitial access hole.

It is recommended that the technique be subjected to further in vitro and in vivo research but until that time practitioners may wish to see whether they find this technique a success.

\section{SUMMARY}

A minimal invasive, highly aesthetic, clinical technique has been presented which facilitates visualisation of the caries, allows for its removal and preserves the contour of the tooth while establishing good contact.
With special thanks: illustrations: Medee Art, www.medeeart.co.uk; dental photography: Ute Fritsche.

1. Roeters F J, Opdam N J, Loomans B A. The amalgam-free dental school. J Dent 2004; 32: 371-377.

2. Soares P V, Santos-Filho P C, Martins L R, Soares $J$ J. Influence of restorative technique on the biomechanical behaviour of endodontically treated maxillary premolars. Part 1: fracture resistance and fracture mode. J Prosthet Dent 2008; 99: 30-37.

3. Peumans M, Van Meerbeek B, Asscherickx K, Simon $S$ et al. Do condensable composites help to achieve better proximal contacts? Dent Mater 2001; 17: 533-541.

4. González-López S, Bolaños-Carmona M V, Navajas Rodríguez de Mondelo J M. Individualized wedge. Oper Dent 2006; 31: 390-393.

5. El-Badrawy W A, Leung B W, El-Mowafy O, Rubo $\mathrm{J} H$, Rubo M H. Evaluation of proximal contacts of posterior resin composite restorations with 4 placement techniques. J Can Dent Assoc 2003; 69: 162-167.

6. Francci C, Loguercio A D, Reis A, Carrilho M R. A novel filling technique for packable composite resin in Class II restorations. J Esthet Restor Dent 2003; 14: 149-157.

7. Loomans B A, Opdam N J, Roeters J F, Bronkhorst E M, Plasschaert A J. Influence of resin composite resin consistency and placement technique on proximal contact tightness of Class II restorations. J Adhes Dent 2006: 8: 305-310.

8. Loomans B A, Opdam N J, Roeters F J, Bronkhorst E M, Burgersdijk R C. Comparison of proximal contacts of Class II resin resin composite restorations in vitro. Oper Dent 2006; 31: 688-693.

9. Loomans B A, Roeters F J, Opdam N J, Kuijs R H. The effect of proximal contour on marginal ridge fracture of class II composite resin restorations. J Dent 2008; 36: 828-832.

10. Loomans B A, Opdam N J, Roeter F J, Bronkhorst E M, Huysmans M C. Restoration techniques and marginal overhangs in class II composite resin restorations. J Dent 2009; 37: 712-717.

11. Loomans B A, Opdam N J, Roeters F J, Bronkhorst $E$ M, Plasschaert $A J$. The long-term effect of a composite resin restoration on proximal contact tightness. J Dent 2007; 35: 104-108.

12. Loomans B A, Opdam N J, Roeters F J, Bronkhorst $\mathrm{E} M$ et al. A randomized clinical trial on proximal contacts of posterior resin composites. J Dent 2006; 34: 292-297.

13. Mount $G J$. An atlas of glass ionomer cements. A clinical guide, 3rd ed. Chapter 2 pp 44-49. London: Martin Dunitz, 1990.

14. Massler M. Changing concepts in the treatment of carious lesions. Br Dent J 1967; 123: 547-548.

15. Feilzer A J, De Gee A J, Davidson C L. Setting stress in resin composite in relation to configuration of the restoration. J Dent Res 1987; 66: 1636-1639.

16. Hasselrot L. Tunnel restorations. A $3 \frac{1}{2}$ year follow up study of class I and class II tunnel restorations in permanent and primary teeth. Swed Dent J 1993; 17: 173-182.

17. Jones S E. The theory and practice of internal 'tunnel' restorations: a review of the literature and observations on clinical performance over eight years in practice. Prim Dent Care 1999; 3: 93-100.

18. Strand G V, Tveit A B, Gjerdet N I, Eide G E. Marginal ridge strength of teeth with tunnel preparations. Int Dent J 1995; 45: 117-123.

19. Wilkie R, Lidums A, Smales D. Class II glass ionome cermet tunnel, resin sandwich and amalgam restorations over 2 years. Am J Dent 1993; 40: 181-184.

20. Ratledge D K, Kidd E A, Treasure E T. The tunne restoration. Br Dent J 2002; 193: 501-506.

21. Strydom C. Handling protocol of posterior resin composites - part 3: matrix systems. SADJ 2006; 61: 18, 20-21.

22. Yong $W$, Zhang $R \mathrm{Q}$. A clinical study of Palodent posterior teeth matrix systems. Hua Xi Kou Qiang Yi Xue Za Zhi 2009; 27: 44-48.

23. Ash M. Wheeler's atlas of tooth form, $5^{\text {th }} \mathrm{ed} . \mathrm{pp}$ 67-68. Philadelphia: WB Saunders Co, 1984. 